\title{
Basic Experimental Studies of the Magnetic Field from Electromagnetic Sources Immersed in a Semi-Infinite Conducting Medium
}

\author{
Martin B. Kraichman ${ }^{1}$
}

(July 20, 1959)

\begin{abstract}
Using electromagnetic sources, consisting of various dipoles and loops immersed in a concentrated sodium chloride solution, measurements were made verifying the magnetic field propagation equations in air, derived previously by several authors. The receiver was farther away from the source than a wavelength in the conducting medium, but much closer than a wavelength in air.

An expression is derived giving the value of the magnetic field in air due to a rectangular loop with a horizontal axis by assuming the loop to consist of two electric dipoles corresponding to the horizontal members. Experimental data verifying this expression are presented.

Also, using submerged electric dipoles, measurements were made of the magnetic field in air which show that the field is determined solely by the current in the horizontal radiating wires of the dipoles.
\end{abstract}

\section{Introduction}

The purpose of the experimental work reported herein is to afford a better understanding of the electromagnetic propagation phenomena involved in radiation from sources immersed in a conducting medium.

Theoretical treatments of the propagation problem have been given by several authors, Tai [1], ${ }^{2}$ Moore [2], Von Aulock [3], Wait [4], Wait and Campbell [5], Lien [6], and Baños and Wesley [7]. The important propagation path was shown to occur mainly through the air above the interface. The field along the direct path through the conducting medium, from source to receiver, suffered an exponential attenuation with the distance and was, therefore, negligible for all but very short distances.

\section{Scope of Work}

The experimental work undertaken at the U.S. Naval Ordnance Laboratory (NOL) consisted in checking the magnetic field propagation equations in air for the horizontal range, where the receiver is farther away from the source than a wavelength in the conducting medium, but much closer than a wavelength in air. Also, experiments were performed to determine the localization of the field source; i.e., whether the currents in the dipole could

1 U.S. Naval Ordnance Laboratory, White Oak, Silver Spring, Md.

2 Figures in brackets indicate the literature references at the end of this paper. be considered as the sole contributors or whether currents in the conducting medium must also be considered in any discussion of the field.

\section{Theory}

In attempting to verify the propagation equations, it was decided to measure the magnetic field components using a shielded loop. This avoids grounding difficulties inherent in the use of an electric antenna. Also, because of the continuity of the magnetic field between air and salt water, measurements made at the surface in air will be valid just below the surface in the conducting medium.

The magnetic field propagation equations for a submerged dipole are given below in cylindrical coordinates using the notation of Baños and Wesley [7]. The cylindrical coordinate system and the position of the dipole are shown in figure 1 . It is understood that the time factor $e^{-i \omega t}$ is to be applied to all field components.

$$
\begin{aligned}
\text { for } & K_{2} \rho \ll 1<\left|K_{1} \rho\right|, K_{2} z \ll 1, \text { and } \frac{z}{\rho}<1, \\
H \rho_{2} & =\frac{i I l \sin \phi}{\pi K_{1} \rho^{3}} e^{i K_{1} h} \\
H \phi_{2} & =\frac{-i I l \cos \phi}{2 \pi K_{1} \rho^{3}} e^{i K_{1} h} \\
H z_{2} & =\frac{-i I l \sin \phi}{2 \pi K_{1} \rho^{3}}\left(\frac{3}{i K_{1} \rho}\right)\left(1-i K_{1} z\right) e^{i K_{1} h},
\end{aligned}
$$




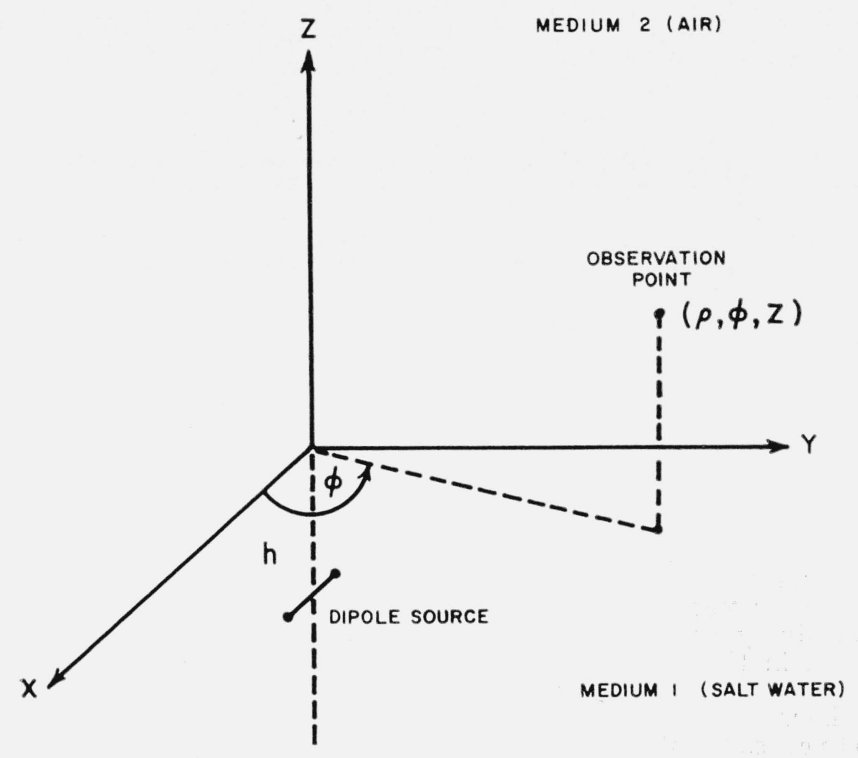

Figure 1. Submorged dipole referred to cylindrical coordinate system.

where

$$
\begin{aligned}
& \omega=\text { angular frequency, } \\
& \mu_{0}=\text { permeability of free space, } \\
& \epsilon_{0}=\text { permittivity of free space, } \\
& \sigma=\text { conductivity of medium } 1 \text { (salt solution), } \\
& K_{1}=\text { propagation constant for plane homo- } \\
& l=\text { dipole length, } \\
& \left.\begin{array}{l}
\rho \\
\phi \\
z
\end{array}\right\}=\text { cylindrical coordinates, } \\
& \mathrm{h}=\text { depth of dipole source. }
\end{aligned}
$$

If a traverse is made parallel to the $x$-axis with the radiating dipole positioned along the axis, a null is found for $\mathrm{Hy}_{2}$. For each traverse the null lies along a line making an angle of approximately $35^{\circ}$ with the $x$-axis. This may be derived from the propagation equations as follows:

$$
\begin{gathered}
H y=H \rho \sin \phi+H \phi \cos \phi . \quad \text { For } H y=0, \\
H \rho \sin \phi=-H \phi \cos \phi .
\end{gathered}
$$

Using expressions for $H_{\rho}$ and $H \phi$ from eq (1) and (2),

or

$$
\frac{i I l \sin ^{2} \phi}{\pi K_{1} \rho^{3}} e^{i K_{1} h}=\frac{i I l \cos ^{2} \phi}{2 \pi K_{1} \rho^{3}} e^{i K_{1} h}
$$

$$
\tan ^{2} \phi=\frac{1}{2}
$$

Thus $\quad \phi=\tan ^{-1} \frac{1}{\sqrt{2}} \cong 35^{\circ} 16^{\prime}$.

In order to verify the absolute value of the field given by eq (1), the expression for the radial component must be modified for calculation in the following way.

If $\quad K_{1}=\left(i \omega \mu_{0} \sigma\right)^{\frac{1}{2}}=i^{\frac{1}{2}} \sqrt{2} \beta$, where $\beta=\left(\frac{\omega \mu_{0} \sigma}{2}\right)^{\frac{1}{2}}$,

then

$$
H \rho_{2}=\frac{i^{\frac{1}{2}} I l \sin \phi}{\pi \sqrt{2} \beta \rho^{3}} e^{i^{\frac{3}{2}} \sqrt{2} \beta h .}
$$

Also since $i^{\frac{1}{2}}=e^{i \frac{\pi}{4}}$ and $i^{\frac{3}{2}}=-\frac{1}{\sqrt{2}}(1-i)$ we get that

$$
H \rho_{2}=\left[\frac{I l}{\pi \sqrt{2} \beta} \frac{\sin \phi}{\rho^{3}} e^{-\beta h}\right] e^{i\left(\beta h+\frac{\pi}{4}\right)} .
$$

The bracketed quantity in eq (5) gives the modulus of the radial magnetic field in air.

Equation (3) may be modified in a similar manner to yield an expression for the vertical magnetic field component.

$$
\begin{aligned}
H z_{2}=\left[\frac{3}{4} \frac{I l \sin \phi}{\pi \beta^{2} \rho^{4}} e^{-\beta \hbar}\right] e^{i\left(\beta h+\frac{\pi}{2}\right)} \\
-\left[\frac{3 \sqrt{2} I l \sin \phi}{4} \pi \frac{z}{\rho^{4}} e^{-\beta h}\right] e^{i\left(\beta h+\frac{5}{4} \pi\right)} .
\end{aligned}
$$

If

and

$$
r_{1}=\frac{3}{4} \frac{I l \sin \phi}{\pi \beta^{3} \rho^{4}} e^{-\beta h}
$$

$$
r_{2}=\frac{-3 \sqrt{2}}{4} \frac{I l \sin \phi}{\pi \beta} \frac{z}{\rho^{4}} e^{-\beta h}
$$

then

$$
\begin{aligned}
H z_{2} & =\left[r_{1} e^{i \frac{\pi}{2}}+r_{2} e^{i \frac{5}{4}}\right] e^{i \beta h} \\
& =\left[\frac{-r_{2}}{\sqrt{2}}+i\left(r_{1}-\frac{r_{2}}{\sqrt{2}}\right)\right] e^{i \beta h} \\
H z_{2} & =\left[\frac{r_{2}^{2}}{2}+\left(r_{1}-\frac{r_{2}}{\sqrt{2}}\right)^{2}\right]^{\frac{1}{2}} e^{i \beta h+\theta}
\end{aligned}
$$

where

$$
\theta=\tan ^{-1} \frac{r_{2}-\sqrt{2} r_{1}}{r_{2}}
$$

The bracketed quantity in eq ( 7 ) gives the modulus of the vertical component of the magnetic field in air.

In calculating the magnetic field due to a rectangular loop with a horizontal axis, the loop may be considered as being composed of horizontal and vertical electric dipoles of length corresponding to the horizontal and vertical members of the loop, respectively. 
It has been shown by Von Aulock [3] that the magnetic field due to a vertical dipole attenuates exponentially with the distance from the surface above the dipole to the point of observation in the conducting medium. This results in a negligible contribution for any reasonable distance of observation from the dipole. Furthermore, the boundary conditions require that the magnetic field be continuous across the surface of the conducting medium. Thus, if measurements are made above the surface of the conducting medium. there should be no contribution to the field in air due to a vertical dipole. The field due to the rectangular loop may then be calculated on the basis of two horizontal members through which the excitation current is oppositely directed.

Considering the radial field, we get from eq (5)

$$
\begin{aligned}
H \rho_{2} & =\left[\frac{I l}{\pi \sqrt{2} \beta} \frac{\sin \phi}{\rho^{3}} e^{-\beta h}\right] e^{i\left(\beta h+\frac{\pi}{4}\right)} \\
H^{\prime} \rho_{2} & =\left[\frac{I l}{\pi \sqrt{2} \beta} \frac{\sin \phi}{\rho^{3}} e^{-\beta h^{\prime}}\right] e^{i\left(\beta h^{\prime}+\frac{\pi}{4}\right)} .
\end{aligned}
$$

Combining eq (9) and (10)

$$
H \rho_{2}+H^{\prime} \rho_{2}=\frac{I l \sin \phi}{\pi \sqrt{2} \beta \rho^{3}} e^{i \frac{\pi}{4}}\left[e^{-\beta h+i \beta h}-e^{-\beta h^{\prime}+i \beta h^{\prime}}\right]
$$

or

$$
\begin{aligned}
& H \rho_{2}+H^{\prime} \rho_{2} \\
& =\frac{I l \sin \phi}{\pi \sqrt{2} \beta \rho^{3}}\left[e^{-2 \beta h}+e^{-2 \beta h^{\prime}}-2 e^{-\beta\left(h+h^{\prime}\right)} \cos \beta\left\{\left(h-h^{\prime}\right)\right\}\right]^{\frac{1}{2}} e^{i\left(\theta+\frac{\pi}{4}\right)}
\end{aligned}
$$

where

$$
\theta=\tan ^{-1} \frac{e^{-\beta h} \sin \beta h-e^{-\beta h^{\prime}} \sin \beta h^{\prime}}{e^{-\beta h} \cos \beta h-e^{-\beta h^{\prime}} \cos \beta h^{\prime}}
$$

The expression for the field of a rectangular loop given by eq (11) agrees with the integrated expression given by Von Aulock [3] for the field of a small coil with a horizontal axis.

\section{Experimental Facilities}

Rather than attempt a full scale verification of the propagation equations at sea, a reduced scale technique was adopted. This was done for a number of reasons. First, the cost of such an operation would be reduced considerably. Second, a land facility would permit wider exploration of experimental variables under more controlled conditions. Third, a facility on the base could readily be used for future investigations.

After balancing cost factors against convenience, the facility constructed consisted of an excavation 20 by $40 \mathrm{ft}$ at the surface, $8-\mathrm{ft}$ deep, with sides sloping at an angle of $45^{\circ}$. The sides and bottom were waterproofed with tar-impregnated felt.
A frequency of approximately $300 \mathrm{kc}$ was chosen. This gave a wavelength of about $4 \mathrm{ft}$ in a $20 \mathrm{mho} / \mathrm{m}$ solution. The pool dimensions in terms of water wavelengths then became 10 wavelengths long by 5 wavelengths wide. The 8 -ft depth permitted simulation of an infinite medium for all practical purposes since the placement of a source at a 4 -ft depth removed it a distance of one wavelength from all interfaces with an accompanying attenuation of approximately $55 \mathrm{db}$.

The sides of the pool were lined at the top with $1-\mathrm{ft}$ wide strips of copper sheeting which were grounded in order to provide continuity between the conducting medium and ground. A satisfactory ground was attained by connecting the sheeting by means of braid to copper rods driven into the earth.

The pool was then filled with a 26 percent solution of sodium chloride giving a conductivity of approximately $20 \mathrm{mho} / \mathrm{m}$ for a temperature of $20^{\circ} \mathrm{C}$.

A General Electric Navy Model TCU-2 transmitter fed the dipole and was operated at 296-kc continuous wave. The transmitter was placed in a double wall screen room located $35 \mathrm{ft}$ from the dipole. To match the $100-\mathrm{ohm}$ transmitter output to the approximately $1-\mathrm{ohm}$ dipole, a transformer with an 11-to-1 turns ratio was used. The lead to the dipole consisted of $95-\mathrm{ohm}$ twinax cable, RG $22 / \mathrm{U}$, with shield grounded, encased in a copper tube which was buried in the earth and which lead directly into the conducting medium. The twinax cable in the conducting medium was connected to the transformer housed in a brass can which was supported at a height of $4 \mathrm{ft}$ above the bottom of the pool. Electric dipoles consisting of insulated wire with silver-plated steel ball terminations were used. Dipole current was measured with a calibrated vacuum thermocouple housed in the brass can and connected in the secondary circuit. Single turn rectangular loops of insulated wire were also used as radiation sources. Magnetic field intensity was. measured in air with a Radio 'Test Set AN/PRM-1.

\section{Results}

The dipoles and loops used as radiating sources are listed below. After a description of each source are listed the depth of immersion, the current fed to the source, the conductivity of the dissipative medium, and the component of magnetic field measured.

A. Horizontal 4-in. dipole; $h=2 \frac{1}{8}$ in.; $I=3.77 \mathrm{amp}$; $\sigma=18.2 \mathrm{mho} / \mathrm{m}$; measured $H_{\rho}$.

B. Horizontal 4 -in. dipole; $h=2 \frac{1}{8}$ in.; $I=3.77 \mathrm{amp}$; $\sigma=18.2 \mathrm{mho} / \mathrm{m}$; measured $H \phi$.

C. Horizontal 4 -in. dipole; $h=6 \frac{1}{8}$ in.; $I=3.77 \mathrm{amp}$; $\sigma=18.2 \mathrm{mho} / \mathrm{m}$; measured $H \rho$.

D. Horizontal 4 -ft dipole; $h=2 \frac{1}{8}$ in. ; $I=1.03 \mathrm{amp}$; $\sigma=17.9 \mathrm{mho} / \mathrm{m}$; measured $H \rho$.

E. Horizontal 4 -ft dipole; $h=38 \frac{1}{8}$ in.; $I=1.26 \mathrm{amp}$; $\sigma=16.1 \mathrm{mho} / \mathrm{m}$; measured $H \rho$.

F. Horizontal $3-\mathrm{ft}$ dipole; $h=4$ in.; $Z=33 \frac{1}{2}$ in.; $I=1.10 \mathrm{amp} ; \sigma=18.2 \mathrm{mho} / \mathrm{m}$; measured $\mathrm{Hz}$. 
G. Single turn 4-in. square loop with axis horizontal; $h=2$ in.; $h^{\prime}=6$ in.; $I=3.77 \mathrm{amp} ; \sigma=18.2 \mathrm{mho} / \mathrm{m}$ measured $H_{\rho}$.

H. Single turn 4 -ft by 3 -ft loop with horizontal axis (4-ft top and bottom members); $h=2$ in.; $\mathrm{h}^{\prime}=38$ in.; $I=1.03 \mathrm{amp} ; \sigma=16.1 \mathrm{mho} / \mathrm{m}$ measured $H \rho$.

I. U-Shape 4-in. square dipole; $h=6 \frac{1}{8}$ in.; $I=3.77$ amp; $\sigma=18.2 \mathrm{mho} / \mathrm{m}$; measured $H \rho$.
All measurements with the above sources were made at a frequency of $296 \mathrm{kc}$.

Measured data are given in table 1 for the various sources. These data are compared in table 2 , which shows the fall off and the experimental and calculated field values at $15 \mathrm{ft}$ from each source. The calculated values were obtained by substitution into the appropriate eq (5), (7), or (11).

TABLE 1. Measured magnetic field data using various radiating sources

Distance from dipole in feet

\begin{tabular}{|c|c|c|c|c|c|c|c|c|c|}
\hline & & & & & & & & & \\
\hline & $A$ & $B$ & $C$ & $D$ & $E$ & $F$ & $G$ & $H$ & $I$ \\
\hline $\begin{array}{l}9 \\
11 \\
13 \\
13_{1} \\
15\end{array}$ & $\begin{array}{l}\text { 54. } 9 \\
\text { 30. } 8 \\
\text { 21. } 6 \\
\text { 14. } 7 \\
\text { 10. } 0\end{array}$ & $\begin{array}{r}\text { 28. } 1 \\
\text { 16. } 3 \\
9.37 \\
6.82 \\
\text { 5. } 08\end{array}$ & $\begin{aligned} 33 . & 5 \\
20 . & 4 \\
13 . & 4 \\
8 . & 70 \\
6 . & 15\end{aligned}$ & $\begin{array}{l}70.2 \\
47.5 \\
34.8\end{array}$ & $\begin{array}{l}\text { 3. } 95 \\
\text { 2. } 41 \\
\text { 1. } 55 \\
\text { 1. } 07 \\
\text { 0. } 682\end{array}$ & $\begin{array}{l}\text { 40. } 1 \\
\text { 22. } 1 \\
\text { 13. } 4 \\
\quad 9.03\end{array}$ & $\begin{aligned} & 30 . 1 \\
& 18.1 \\
& 11.5 \\
& 7.63 \\
& 5.55\end{aligned}$ & $\begin{array}{l}83.6 \\
55.0 \\
40.1\end{array}$ & $\begin{aligned} \text { 33. } & 4 \\
20 . & 4 \\
12 . & 8 \\
8 . & 69 \\
6 . & 02\end{aligned}$ \\
\hline $\begin{array}{l}19 \\
21 \\
23 \\
25 \\
25 \\
29\end{array}$ & $\begin{array}{l}\text { 6. } 89 \\
5.49 \\
\text { 4. } 35 \\
\text { 3. } 55 \\
\text { 2. } 51 \\
\text { 2. } 14\end{array}$ & $\begin{array}{l}3.75 \\
2.54 \\
2.41 \\
\text { 1. } 91 \\
\text { 1. } 51 \\
1.20\end{array}$ & $\begin{array}{l}\text { 4. } 48 \\
\text { 3. } 41 \\
2.54 \\
\text { 2. } 07 \\
1.61 \\
1.34\end{array}$ & $\begin{array}{r}25.4 \\
19.4 \\
14.7 \\
11.7 \\
8.90 \\
6.82\end{array}$ & $\begin{array}{r}.482 \\
.335 \\
.241 \\
.174 \\
- \\
\end{array}$ & $\begin{array}{l}\text { 6. } 02 \\
\text { 4. } 01 \\
\text { 3. } 01 \\
\text { 2. } 17 \\
1.67 \\
1.27\end{array}$ & $\begin{array}{l}\text { 4. } 01 \\
\text { 3. } 01 \\
2.34 \\
1.87 \\
1.61 \\
1.20\end{array}$ & $\begin{array}{r}31.4 \\
22.7 \\
17.4 \\
13.4 \\
10.7 \\
8.56\end{array}$ & $\begin{array}{l}\text { 4. } 35 \\
\text { 3. } 41 \\
\text { 2. } 52 \\
\text { 2. } 01 \\
\text { 1. } 58 \\
\text { 1. } 32\end{array}$ \\
\hline
\end{tabular}

TABLE 2. Comparison of magnetic field data from various radiating sources

\begin{tabular}{|c|c|c|c|}
\hline Source & $\begin{array}{l}\text { Measured } \\
\text { fall off }\end{array}$ & $\begin{array}{c}\text { Measured } \\
\text { field at } 15 \\
\mathrm{ft} \\
\text { amp/m } \times 10^{5}\end{array}$ & $\begin{array}{c}\text { Calculated } \\
\text { field at } 15 \\
\mathrm{ft} \\
\mathrm{amp} / \mathrm{m} \times 10^{5}\end{array}$ \\
\hline $\begin{array}{l}A_{\ldots} \\
B_{-} \\
C_{-} \\
D_{-} \\
E_{-} \ldots\end{array}$ & $\begin{array}{l}\text { 2. } 9 \\
\text { 3. } 1 \\
\text { 3. } 0 \\
\text { 3. } 0 \\
\text { 3. } 3\end{array}$ & $\begin{aligned} 14.7 \\
6.82 \\
8.70 \\
47.5 \\
1.07\end{aligned}$ & $\begin{aligned} 15.3 \\
7.65 \\
9.59 \\
50.7 \\
1.03\end{aligned}$ \\
\hline $\begin{array}{l}F_{\ldots} \ldots \ldots \\
G_{\ldots} \\
H_{\ldots} \\
I_{\ldots} \ldots \ldots\end{array}$ & $\begin{array}{l}\text { 3. } 9 \\
\text { 3. } 0 \\
\text { 3. } 0 \\
\text { 3. } 0\end{array}$ & $\begin{aligned} \text { 13. } & 4 \\
\text { 7. } & 63 \\
55 . & 0 \\
8 . & 69\end{aligned}$ & $\begin{aligned} & \text { 10. } 2 \\
& 8 . 20 \\
& 55.9 \\
& 9 . 37\end{aligned}$ \\
\hline
\end{tabular}

The data from sources $A$ and $B$ are plotted in figure 2. This plot shows the inverse cube fall off for $H_{\rho_{2}}$ and $H \phi_{2}$ and the 6-db factor between them.

An examination of the results given in table 2 shows good agreement with theory both with respect to fall off and absolute value. All experimental field values except those from source $F$ were within $1 \mathrm{db}$ of the calculated values. The field value from source $F$ was within $2.4 \mathrm{db}$ of the calculated value.

The nulls of figure 3 were found to occur at $35^{\circ} 32^{\prime}$ for the 20 -ft traverse and $37^{\circ} 11^{\prime}$ for the 55 - $\mathrm{ft}$ traverse, thus verifying eq (4).

From the experimental and calculated results presented, it appears that the field is determined solely by the current in the horizontal radiating wires of the dipole or loop. Since this conclusion is not par-

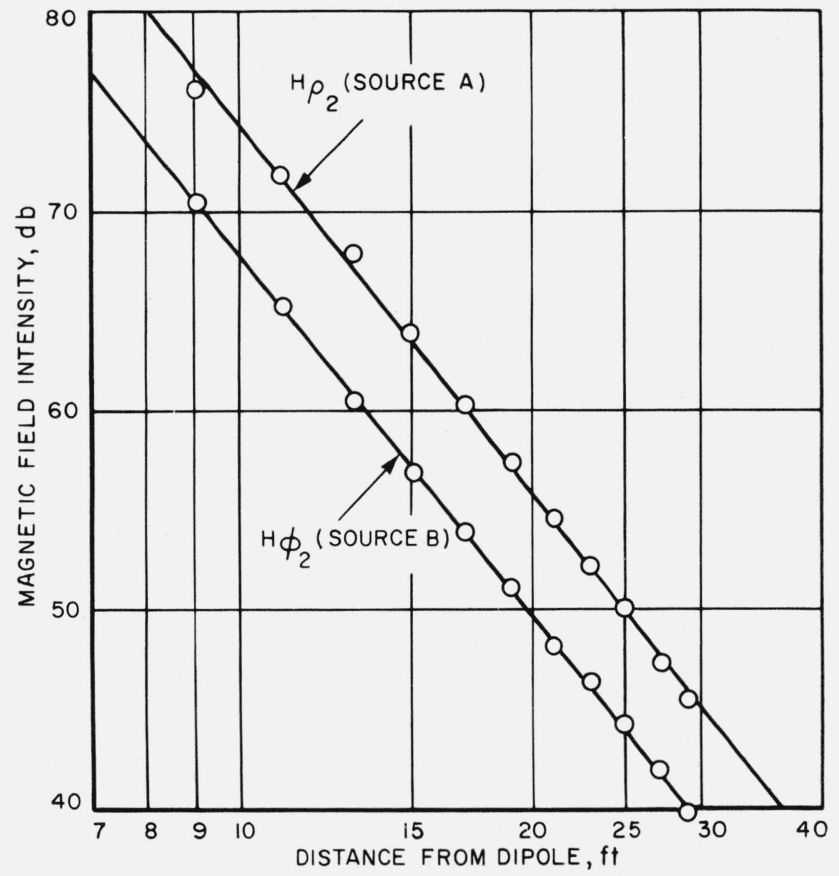

Figure 2. Fall off of horizontal magnetic field components. Reference level: $9.65 \times 10^{-8} \mathrm{amp} / \mathrm{m}$

ticularly obvious, the following experiment was performed to more clearly determine whether or not this was actually the case.

Sources $A, C$, and $I$ were placed below the surface of the conducting medium as shown in figure 4 , and the field radiated from each was measured. The 

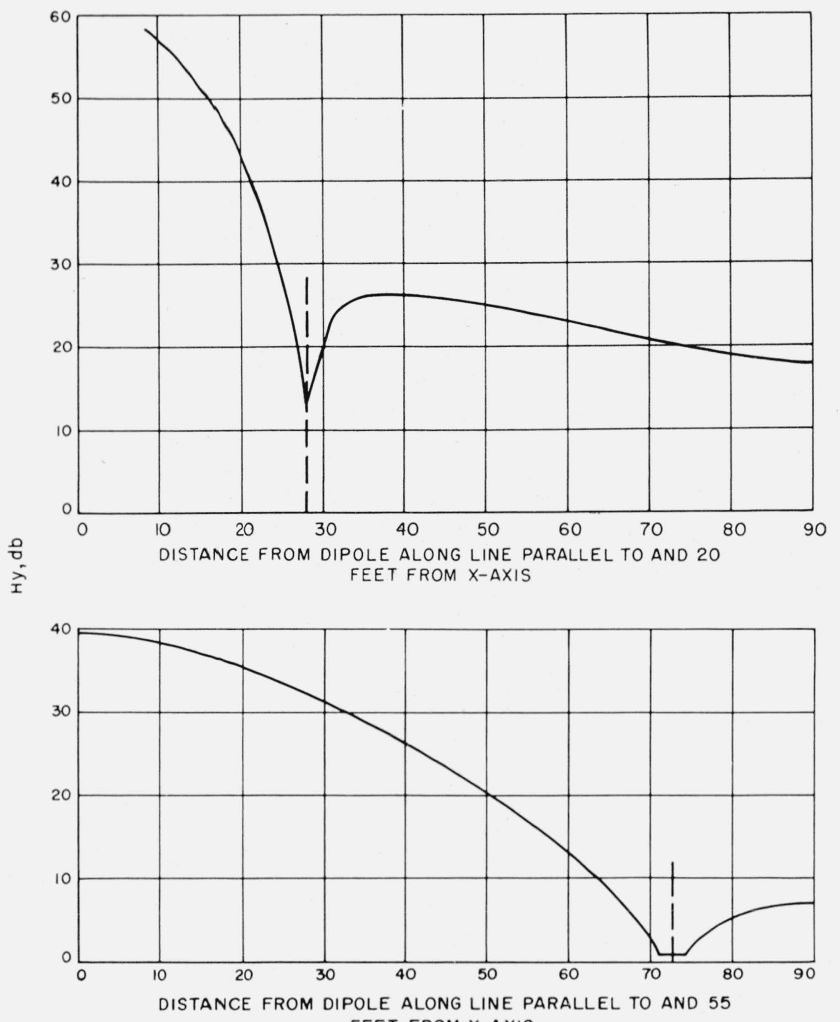

FEET FROM X-AXIS

Figure 3. Magnetic field plot of $\mathrm{Hy}$ from traverse parallel to dipole axis.

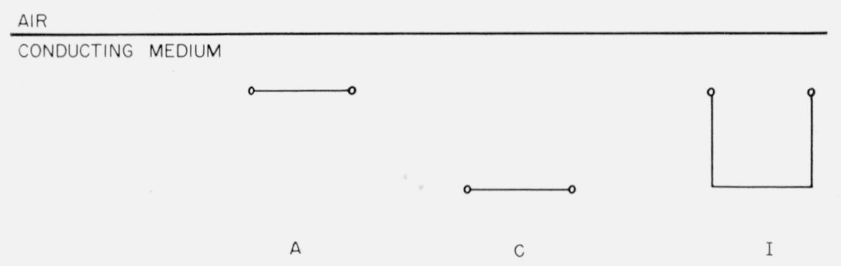

Figure 4. Positions of radiating sources for determining localization of current moment. results given in tables 1 and 2 show that source $I$ was equivalent to source $C$ and not to source $A$. Thus, the currents in the horizontal wires of the dipoles determine the value of the radiated field.

\section{Conclusions}

On the basis of the experimental results described, the following conclusions may be drawn:

(a) The magnetic field propagation eq (1), (2), and (3), for the range therein stated, are valid.

(b) For calculating the magnetic field of a dipole using these equations, the current moment may be considered as due only to the currents in the horizontal wires forming the dipole.

(c) Calculations for a rectangular loop with horizontal axis may be made on the basis of the loop consisting of two horizontal dipoles carrying current in opposite directions and properly phased according to depth in the conducting medium.

\section{References}

[1] C. T. Tai, Hertzian dipole immersed in a dissipative medium, Cruft Lab. Rept. No. 21, Harvard Univ. (1947).

[2] R. K. Moore, The theory of radio communication between submerged submarines, Ph. D. Thesis, Cornell Univ. (1951)

[3] W. Von Aulock, Low frequency electromagnetic dipole fields in a semi-infinite conductor, Navy Dept., Bureau of Ships, Minesweeping Sec., Tech. Rept., No. 104 (1952).

[4] J. R. Wait, Magnetic dipole antenna in a conducting medium, Proc. I.R.E., 40, 1244-45 (1952).

[5] J. R. Wait and L. L. Campbell, The fields of an oscillating magnetic dipole immersed in a semi-infinite conducting medium, J. Geophys. Research, 58, 167-168 (1953).

[6] R. H. Lien and J. R. Wait, Radiation from a horizontal dipole in a semi-infinite dissipative medium, J. Appl. Phys. 24, 1-5, 958-959 (1953).

[7] A. Baños, Jr., and J. P. Wesley, The horizontal electric dipole in a conducting half-space, Pt. I, Univ. Calif., Marine Phys. Lab., Scripps Inst. Oceanography, SIO Ref. 53-33 (Sept. 1953); Pt. II, SIO Ref. 54-31 (Aug. 1954).

Boulder, Colo.

(Paper 64D1-33) 